\title{
Guiding-Center-Drift Resonance in a Periodically Modulated Two-Dimensional Electron Gas
}

\author{
C. W. J. Beenakker \\ Philips Research Laboratories, 5600 JA Eindhoven, The Netherlands
}

(Received 27 January 1989)

\begin{abstract}
A theory is developed for the recently discovered magnetoresistance oscillations in a two-dimensional electron gas subject to a weak periodic potential. The effect is explained as a resonance between the periodic cyclotron orbit motion and the oscillating $\mathbf{E} \times \mathbf{B}$ drift of the orbit center induced by a potential grating.
\end{abstract}

PACS numbers: $71.25 . \mathrm{Hc}, 72.20 . \mathrm{My}, 73.50 . \mathrm{Jt}$

Spatially modulated semiconductor structures in a magnetic field have unusual properties due to the interplay of the two independent periodicities of the modulation and the cyclotron orbit. Recently, Weiss, von Klitzing, Ploog, and Weimann' 'discovered a striking manifestation of this interplay in the magnetoresistance of a two-dimensional electron gas (2DEG) subject to a weak periodic potential variation in one direction (a potential grating). At low magnetic fields $B$ (perpendicular to the $2 D E G)$ an oscillation periodic in $1 / B$ was observed in the resistance, reminiscent of the Shubnikov-de Haas (SdH) oscillations at higher fields-but with a different periodicity and a much weaker temperature dependence. The new periodicity was found to be given by the condition that the cyclotron orbit radius $R \equiv m v_{F} / e B$ is an integer multiple for the modulation period $a\left(v_{F}\right.$ is the Fermi velocity). Weiss et al. remarked that this periodicity corresponds to $\mathrm{SdH}$ oscillations where only electrons within the first Brillouin zone of the modulated structure contribute, but no mechanism was found to support an explanation along these lines.

Theoretically, the transport properties of a periodically modulated 2DEG have been studied with the emphasis on effects originating from the band structure of the lateral superlattice. ${ }^{2}$ In the experiments of Weiss et al., ${ }^{1}$ however, the period $a=0.3-0.4 \mu \mathrm{m}$ is considerably larger than the Fermi wavelength $\lambda_{F} \equiv 2 \pi / k_{F} \sim 50 \mathrm{~nm}$, suggesting a different origin of their effect. Moreover, the weak temperature dependence of the oscillation amplitude indicates that magnetic quantization in Landau levels (responsible for the $\mathrm{SdH}$ oscillations) does not play an important role. These considerations motivated me to look for a semiclassical explanation. I have found that the magnetoresistance oscillation induced by a potential grating is due to a resonance in the $\mathbf{E} \times \mathbf{B}$ drift of the cyclotron orbit (guiding) center. Such resonances are known from plasma physics, ${ }^{3,4}$ and the experiment by Weiss $e t$ al. appears to be the first observation of this phenomenon in the solid state.

This paper consists of two parts. A detailed systematic transport theory is developed, based on the semiclassical Boltzmann equation in the relaxation-time approximation. The analysis is somewhat involved because of the presence of both a magnetic field and an inhomogeneous electric field. Therefore, I first present a simplified physical picture of the resonance mechanism and its effect on the transport properties.

The guiding center $(X, Y)$ of an electron at position $(x, y)$ having velocity $\left(v_{x}, v_{y}\right)$ is given by $X=x-v_{y} / \omega_{c}$, $Y=y+v_{x} / \omega_{x}$, with $\omega_{c} \equiv e B / m$ the cyclotron frequency. The choice of axes is such that the (homogeneous) magnetic field $B$ is in the $z$ direction, perpendicular to the 2DEG, and the potential grating $V(y)$ induces an electric field $E \equiv-d V / d y$ in the $y$ direction (see Fig. 1). The time derivative of the guiding center is $\dot{X}=E(y) / B$, $\dot{Y}=0$, so that its motion is parallel to the $x$ axis. This is the $\mathbf{E} \times \mathbf{B}$ drift. In the case of a strong magnetic field and a slowly varying potential $(R \ll a)$ one may approximate $E(y) \approx E(Y)$ to close the equations for $\dot{X}$ and $\dot{Y}$. This so-called adiabatic approximation cannot be made in the weak-field regime $(R \gtrsim a)$ of interest here. I consider the case of a weak potential, such that $e V_{\mathrm{rms}} / E_{F} \equiv \epsilon \ll 1$, with $V_{\text {rms }}$ the root mean square of $V(y)$ and $E_{F} \equiv \frac{1}{2} m v_{F}^{2}$ the Fermi energy. [Small values of $\epsilon$ on the order of a few percent are obtained experimentally by Weiss et al.,' using the (nonlithographic) technique of holographic illumination.] The guidingcenter drift is then simply superimposed on the unperturbed cyclotron motion. Its time average $v_{\text {drift }}$ is obtained by integrating the electric field along the orbit,

$$
v_{\text {drift }}(Y)=(2 \pi B)^{-1} \int_{0}^{2 \pi} d \phi E(Y+R \sin \phi) .
$$

For $R \gg a$ the field oscillates rapidly, so that only the drift acquired close to the two extremal points $Y \pm R$ does not average out. It follows that $v_{\text {drift }}$ is enhanced or reduced depending on whether $E(Y+R)$ and $E(Y-R)$ have the same or opposite sign (see Fig. 1). Such an oscillatory guiding-center drift has been noticed before in a plasma physical context. ${ }^{3}$ For a sinusoidal potential ${ }^{5}$ $V(y)=V_{\mathrm{rms}} 2^{1 / 2} \sin (2 \pi y / a)$ one easily calculates that for $R \gg a$ the mean square drift on averaging over $Y$ is

$$
\left\langle v_{\text {drift }}^{2}\right\rangle=\left(v_{F} \epsilon\right)^{2}(R / a) \cos ^{2}(2 \pi R / a-\pi / 4) \text {. }
$$

The guiding-center drift by itself leads, for $\omega_{c} \tau \gg 1$, to one-dimensional diffusion with diffusion coefficient $\delta D$ 
(a)
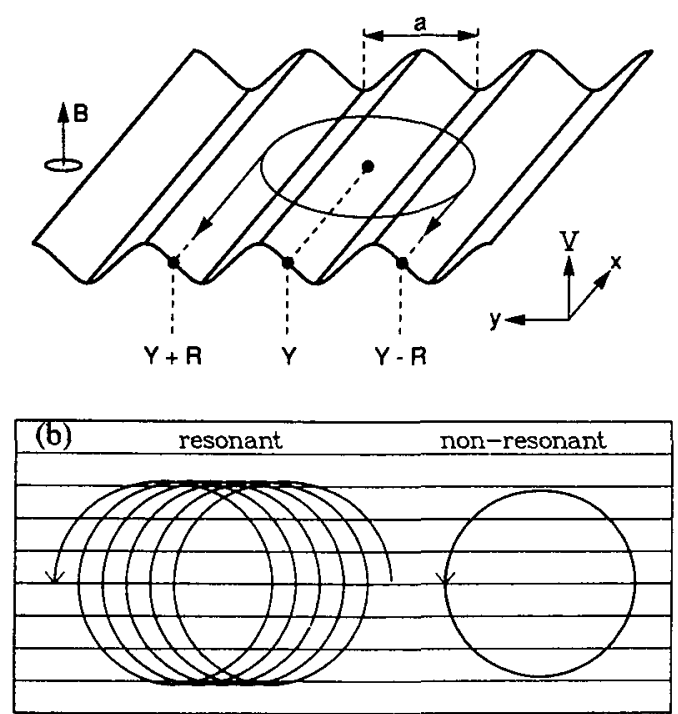

FIG. 1. (a) Potential grating with a cyclotron orbit superimposed. When the electron is close to the two extremal points $Y \pm R$ the guiding center at $Y$ acquires an $\mathbf{E} \times \mathbf{B}$ drift in the direction of the arrows. (The drift along nonextremal parts of the orbit averages out, approximately.) A resonance occurs if the drift at one extremal point reinforces the drift at the other, as shown. (b) Numerically calculated trajectories for a sinusoidal potential $(\epsilon=0.015)$. The horizontal lines are equipotentials at integer $y / a$. On resonance $(2 R / a=6.25)$ the guiding-center drift is maximal; off resonance $(2 R / a=5.75)$ the drift is negligible.

$\tau\left\langle v_{\text {drift }}^{2}\right\rangle$ ( $\tau$ being the scattering time). The term $\delta D$ is an additional contribution to the $x x$ element of the unperturbed diffusion tensor $\mathbb{D}^{0}$, given by $D_{x x}^{0}=D_{y y}^{0}=D_{0}$, $D_{x y}^{0}=-D_{y x}^{0}=-\omega_{c} \tau D_{0}$, with $D_{0} \equiv \frac{1}{2} \tau v_{F}^{2}\left[1+\left(\omega_{c} \tau\right)^{2}\right]^{-1}$.
At this point I assume that for $\omega_{c} \tau \gg 1$ the above contribution $\delta D$ from the guiding-center drift is the dominant effect of the potential grating on the diffusion tensor $D$. A justification of this assumption requires a more systematic analysis of the transport problem, which will be given below in the second part of this paper. Once $\mathbf{D}$ is known, the resistivity tensor $\rho$ follows from the Einstein relation $\rho=\left(1 / N e^{2}\right) D^{-1}$, with $N=4 \pi m / h^{2}$ the density of states (which is energy independent in a 2DEG; $N$ contains a factor of 2 from the spin degeneracy). The unperturbed diffusion tensor $D^{0}$ gives a longitudinal resistivity which is isotropic and $B$ independent, $\rho_{x x}^{0}=\rho_{y y}^{0}=\rho_{0} \equiv h / k_{F} l e^{2}$ (with $l \equiv \tau v_{F}$ the mean free path). The Hall resistance is $\rho_{x y}^{0}=-\rho_{y x}^{0}=\omega_{c} \tau \rho_{0}$. Because of the large off-diagonal components of $\mathbf{D}^{0}$, an additional contribution $\delta D$ to $D_{x x}^{0}$ modifies predominantly the $y y$ component of $\rho$, which is the resistivity to current flowing perpendicular to the grating. To leading order in $\epsilon$ one finds that

$$
\rho_{y y} / \rho_{0}=1+2 \epsilon^{2}\left(l^{2} / a R\right) \cos ^{2}(2 \pi R / a-\pi / 4) .
$$

In the other components of $\rho$ corrections appear which are smaller by a factor $\left(\omega_{c} \tau\right)^{2}$, and are spurious consequences of retaining only the effect on $D_{x x}$ of the potential grating (no such approximation is made below). I defer a discussion on Eq. (1), and show first how this result is borne out by a more detailed and systematic analysis.

The analysis is based on the Boltzmann equation in the relaxation-time approximation, which is the usual level of description in semiclassical transport theory. ${ }^{6}$ I derive the required resistivity tensor by means of the Einstein relation from the diffusion tensor, which itself follows from the (stationary) Boltzmann equation for noninteracting electrons at the Fermi level, ${ }^{7}$

$$
\mathcal{L} f \equiv\left(v(y) u \cdot \frac{\partial}{\partial \mathrm{r}}+\left[v^{\prime}(y) \cos \phi+\omega_{c}\right] \frac{\partial}{\partial \phi}+\frac{1}{\tau}-\int_{0}^{2 \pi} \frac{d \phi}{2 \pi \tau}\right) f(x, y, \phi) \equiv 0 .
$$

Here $f(x, y, \phi)$ is the distribution function for electrons at point $\mathrm{r} \equiv(x, y)$ with velocity direction $\mathrm{u} \equiv(\cos \phi, \sin \phi)$. The magnitude of the velocity $v(y) \equiv v_{F}[1+e V(y) /$ $\left.E_{F}\right]^{1 / 2}$ (with derivative $v^{\prime} \equiv d v / d y$ ) is $y$ dependent because of the potential grating. Short-ranged and isotropic elastic impurity scattering is assumed, leading to a constant scattering time $\tau$. If a solution to Eq. (2) can be found with a constant density gradient $c \equiv\left(c_{x}, c_{y}\right)$ (averaged over one period of the potential), then the diffusion tensor follows from $\mathbf{j}=-\mathbf{D} \cdot \mathbf{c}$, with the particle current density given by $\mathrm{j}=a^{-1} \int_{0}^{a} d y \int_{0}^{2 \pi} d \phi f v u$.

To determine $\mathrm{D}, I$ make a transformation from $f$ to a new unknown $F$, by equating

$2 \pi f \equiv \mathbf{c} \cdot \mathbf{r}-2 v(y)_{v_{F}}{ }^{-2} \mathbf{u} \cdot \mathbb{D}^{0} \cdot \mathbf{c}-F D_{0}\left(\omega_{c} \tau c_{x}+c_{y}\right)$.

Equation (2) is satisfied if $\mathcal{L} F=e E(y) / E_{F}$. Consider the solution $F \equiv F(y, \phi)$ which does not depend on $x$ and is periodic in $y$ with period $a$. The distribution function $f$ then has the required constant average density gradient c, while $F$ does not depend on $c$. The components of $D$ can now immediately be extracted from the expression for $j$ Iwhich is why the transformation (3) was chosen in that way]. Using the Einstein relation to go from $D$ to $\rho$ one finds that only $\rho_{y y}$ is modified by the potential grating,

$$
\begin{aligned}
& \rho_{y y} / \rho_{0}=1+\left[1+\left(\omega_{c} \tau\right)^{2}\right] \frac{K}{1-K}, \\
& K \equiv D_{0} \int_{0}^{a} \frac{d y}{a} \int_{0}^{2 \pi} \frac{d \phi}{2 \pi} \frac{e E(y)}{E_{F}} F(y, \phi) .
\end{aligned}
$$

The other components of $\rho$ remain those of $\rho^{0}$. This is an exact consequence of the Boltzmann equation (2) for arbitrary potential grating.

It remains to evaluate $K$, which for a weak potential 
can be done perturbatively. To first order in $\epsilon, F$ is determined by $\mathcal{L}_{0} F=e E(y) / E_{F}$, where $\mathcal{L}_{0}$ is $\mathcal{L}$ for $V(y) \equiv 0$. This equation can be solved straightforwardly, and for a sinusoidal potential I find

$$
\begin{aligned}
& K=\frac{1}{2}\left[1+\left(\omega_{c} \tau\right)^{2}\right]^{-1}(\epsilon q l)^{2} S(1-S)^{-1}, \\
& S=\sum_{p}^{\infty} J_{p}^{2}(q R)\left[\left(p \omega_{c} \tau\right)^{2}+1\right]^{-1},
\end{aligned}
$$

with $q \equiv 2 \pi / a$. Inserting $K$ into Eq. (4) one finds that to second order in $\epsilon$, and for ${ }^{8} \omega_{c} \tau \gg 1$,

$$
\rho_{y y} / \rho_{0}=1+\frac{1}{2}(\epsilon q l)^{2} J_{0}^{2}(q R)\left[1-J_{0}^{2}(q R)\right]^{-1} .
$$

For $q R \gg 1$, Eq. (6) reduces to Eq. (1), which confirms the main result of the simplified picture given in the first part of this paper.

In Fig. 2 the theoretical $\rho_{y y}$ is compared with the experimental result of Weiss et al. ${ }^{1}$ The parameters $a=382 \mathrm{~nm}, l=12 \mu \mathrm{m}$, and $k_{F}=0.14 \mathrm{~nm}^{-1}$ have been obtained directly from Ref. 1. The experimental amplitude of the periodic potential (which determines the parameter $\epsilon$ ) is not known precisely. In Fig. 2 I have chosen $\epsilon=0.015$ to bring the scale on the vertical axis in agreement with the experimental data. The scale on the horizontal axis does not contain any free parameters, so that the agreement obtained on the position of the resistivity maxima and minima is a significant support for the present theory. As illustrated by the arrows in Fig. 2, the maxima in $\rho_{y y}$ are not at integer $2 R / a$, but shifted somewhat towards lower magnetic fields. This phase shift was noticed experimentally by Weiss et al., and is reproduced quite accurately by Eq. (6). The resonance mechanism for the oscillations presented here predicts a

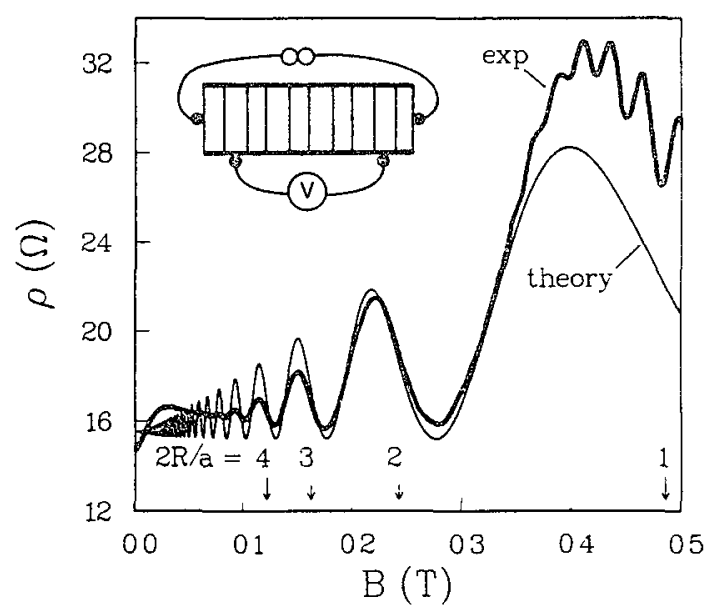

FIG. 2. Magnetic field dependence of the resistivity $\rho_{y y}$ for current flowing perpendicular to the potential grating (see inset). The theoretical curve is from Eq. (6); the experimental curve from Ref. 1. Note the phase shift of the oscillations, as indicated by the arrows at integer $2 R / a$. For $B \gtrsim 0,4 \mathrm{~T}$ the experimental data show the onset of the Shubnikov-de Haas oscillations. relative amplitude of order $\epsilon^{2}\left(l^{2} / a R\right)$, which for a large mean free path can be of order $1-$ even if $\epsilon \ll 1$. This explains the surprising experimental finding that a periodic modulation of the Fermi velocity of order $10^{-2}$ can double the resistivity.

At low magnetic fields the experimental oscillations are damped more rapidly than the theory would predict, and, moreover, a positive magnetoresistance is observed around zero field which is not found here. Part of this disagreement may be due to nonuniformities in the potential grating, which become especially important at low fields when the cyclotron orbit overlaps many modulation periods. At high magnetic fields $B \gtrsim 0.4 \mathrm{~T}$ the experimental data show the onset of $\mathrm{SdH}$ oscillations, which are not described by the present low-field theory in which Landau-level quantization is ignored. Neither $\rho_{x x}$ nor $\rho_{x y}$ are affected by the potential grating in the present theory. Experimentally ${ }^{\prime}$ a weak oscillatory structure is found in the resistivity $\rho_{x x}$ to current flowing parallel to the grating (the Hall resistance $\rho_{x y}$ does not show any oscillations). This remains to be understood.

In summary, both a simplified physical picture and a systematic transport theory have been presented for the recently discovered ${ }^{1}$ magnetoresistance oscillations induced by a potential grating in a 2DEG. It is proposed that this effect is the first example of a new class of magnetotransport phenomena due to guiding-center-drift resonances. In view of the recent developments in ballistic transport, ${ }^{9}$ it is to be expected that more of such plasma physical effects in a 2DEG can be found.

I thank R. R. Gerhardts, K. von Klitzing, and D. Weiss for introducing me to this problem, and G. E. W. Bauer, H. van Houten, and M. F. H. Schuurmans for frequent discussions.

Note added.- Results equivalent to Eq. (1) in the first part of this paper have recently been obtained independently by Gerhardts, Weiss, and von Klitzing ${ }^{10}$ and Winkler, Kotthaus, and Ploog, ${ }^{11}$ who calculated the oscillatory $B$ dependence of the Landau bandwidth. Such oscillatory behavior is indeed a consequence of the oscillatory guiding-center drift considered here. Note, however, that the Landau band quantization (i.e., the discreteness of the band index) is not essential for the occurrence of the magnetoresistance oscillations-which as is demonstrated here follows basically from classical mechanics.

\footnotetext{
${ }^{1}$ D. Weiss, K. von Klitzing, K. Ploog, and G. Weimann, Europhys. Lett. 8, 179 (1989); in The Application of High Magnetic Fields in Semiconductor Physics, edited by G. Landwehr, Lecture Notes in Physics Vol. 177 (Springer-Verlag, Berlin, 1983).

${ }^{2}$ H. Sakaki, K. Wagatsuma, J. Hamasaki, and S. Saito, Thin Solid Films 36, 497 (1976); M. J. Kelly, J. Phys. C 18, 6341 (1985); A. C. Warren, D. A. Antoniadis, H. I. Smith, and J. Melngailis, IEEE Electron. Device Lett. 6, 294 (1985); Y.
} 
Tokura and K. Tsubaki, Appl. Phys. Lett. 51, 1807 (1987); K. Ismail, W. Chu, D. A. Antoniadis, and H. I. Smith, Appl. Phys. Lett. 52, 1071 (1988).

${ }^{3}$ G. Knorr, F. R. Hansen, J. P. Lynov, H. L. Pécseli, and J. J. Rasmussen, Phys. Scr. 38, 829 (1988).

${ }^{4}$ The guiding-center-drift resonance considered here is unrelated to the magnetoacoustic resonance discussed extensively by M. H. Cohen, M. J. Harrison, and W. A. Harrison, Phys. Rev. 117, B937 (1960). The latter effect is the response to a nonconservative electric field, in which the electron can have a net increase in speed per completed cyclotron orbit. In contrast, the effect considered here is induced by a conservative field derived from an electrostatic potential.

${ }^{5}$ The assumption of a sinusoidal potential is presumably a reasonable approximation for the experiments of Ref. 1, but is not essential for the calculations.
${ }^{6} \mathrm{R}$. G. Chambers, in The Physics of Metals, edited by J. M. Ziman (Cambridge Univ. Press, London, 1969), Vol. 1, p. 175.

${ }^{7}$ The Boltzmann equation may look unfamiliar in this form because no derivatives with respect to the magnitude $v$ of the velocity occur. These have been eliminated by means of the equation $\frac{1}{2} m v^{2}-e V(y)-E_{F}$, which expresses the conservation of energy in the absence of inelastic scattering.

${ }^{8}$ The result to order $\epsilon^{2}$ for $\omega_{c} \tau \ll 1$ is $\rho_{y y} / \rho_{0}=1$ $+\frac{1}{2}(\epsilon q l)^{2}\left\{\left[1+(q l)^{2}\right]^{1 / 2}-1\right\}^{-1}$.

${ }^{9}$ Physics and Technology of Submicron Structures, edited by $H$. Heinrich, G. Bauer, and F. Kuchar (Springer-Verlag, Berlin, 1988).

${ }^{10}$ R. R. Gerhardts, D. Weiss, and K. von Klitzing, Phys. Rev. Lett. 62, 1173 (1989).

"R. W. Winkler, J. P. Kotthaus, and K. Ploog, Phys. Rev. Lett. 62, 1177 (1989). 